\title{
A novel sample delivery system based on circular motion for serial crystallography
}

\author{
Feng-Zhu Zhao, Xiao-Qian Jin, Liang-Liang Chen, Da-Chuan Yin* \\ School of Life Sciences, Northwestern Polytechnical University, Xi'an, China. \\ yindc@nwpu.edu.cn
}

Serial crystallography has made remarkable progress since its origin. The advantages of serial crystallography cover the aspects of room-temperature structure determination of the biomacromolecules, micron or sub-micron sized crystal structure determination, as well as time-resolved research. Sample delivery system is one of the key parts of serial crystallography. It is the main limiting factor affecting the application of serial crystallography. In the existing sample delivery technologies, the samples are usually delivered in linear motion. Here we show that the samples can be also delivered using circular motion, which is a novel motion mode never tested before. We report a microfluidic rotating-target sample delivery device, which is characterized by the circular motion of the samples, and verify the performance of the device on the synchrotron radiation facility ${ }^{[1]}$. The microfluidic rotating-target sample delivery device consists of two parts: a microfluidic sample plate and a motion control system. Sample delivery is realized by rotating the microfluidic sample plate containing in-situ grown crystals as shown in Fig. 1. This device offers significant advantages, including a very wide adjustable range of delivery speed, low background noise, and low sample consumption. Using the microfluidic rotatingtarget device, we carried out in-situ serial crystallography experiments with lysozyme and proteinase $\mathrm{K}$ as model samples on the Shanghai Synchrotron Radiation Facility, and performed the structural determination based on the serial crystallographic data. The results showed that the designed device is fully compatible with the synchrotron radiation facility, and the structure determination of proteins is successful using the serial crystallographic data obtained with the device.

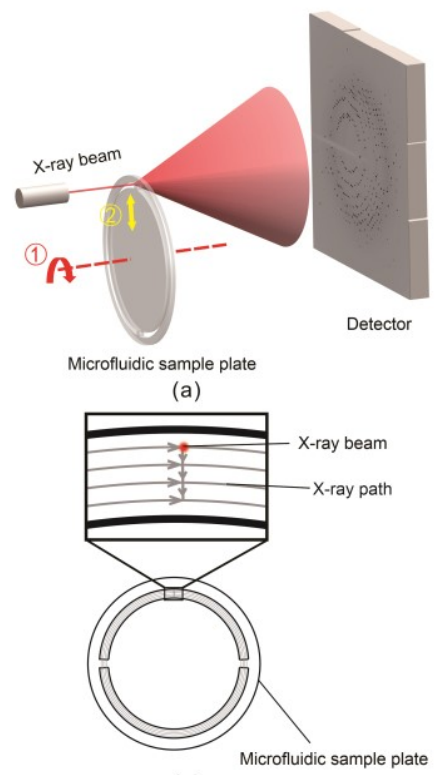

(c)

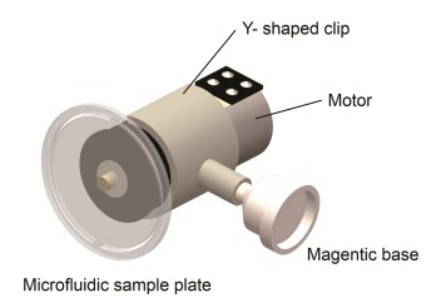

(b)

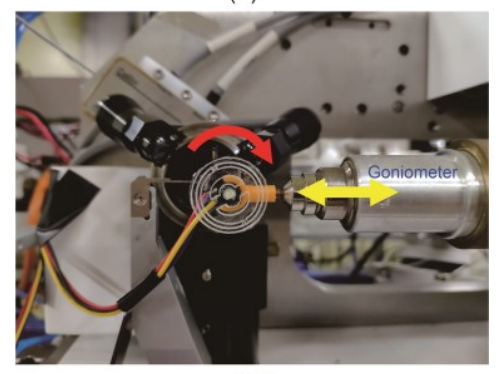

(d)

Figure 1. Diagram of the microfluidic rotating-target device. (a) Principle diagram of the microfluidic rotating-target device. (b) Installation diagram of the motor and microfluidic sample plate. (c) Schematic diagram of the motion control scheme for microfluidic sample plate. (d) Physical picture of the microfluidic rotating-target device.

[1] Zhao, F. Z., Sun, B., Yu, L., Xiao, Q. J., Wang, Z. J., Chen, L. L., Liang, H., Wang, Q. S., He, J. H. \& Yin, D. C. (2020) Lab Chip, 20, 38883898 .

Keywords: serial crystallography; sample delivery system; circular motion; in-situ diffraction; microfluidic

This work was supported by the National Key Research and Development Plan of China (Grant No. 2017YFA0504901), the Innovation Capability Support Program of Shaanxi (Program No. 2020TD-042), the Natural Science Basic Research Plan in Shaanxi Province of China (2019JM-581), the Innovation Foundation for Doctor Dissertation of Northwestern Polytechnical University (CX202060).

Acta Cryst. (2021), A77, C930 